\title{
Elternfaktoren bei Internet- und Computerspielsucht im Jugendalter: Eine Übersicht
}

\author{
Isabel Brandhorst, Tobias Renner und Gottfried Maria Barth \\ Abteilung Psychiatrie, Psychosomatik und Psychotherapie im Kindes- und Jugendalter, Klinik für \\ Psychiatrie und Psychotherapie, Universitätsklinikum Tübingen
}

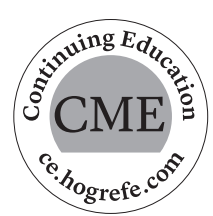

Zusammenfassung: Einleitung: Internetbezogene Störungen (IBS) bei Jugendlichen und jungen Erwachsenen sind eng mit Familienfaktoren verknüpft. Dennoch beziehen wenige Forschungs- und Übersichtsarbeiten die Familie mit ein. Methodik: Die vorliegende Arbeit gibt einen Überblick zu Elternfaktoren, die bei der Entstehung, Aufrechterhaltung und Reduktion einer Symptomatik beteiligt sein können. Hierfür wurden $N=87$ wissenschaftliche Arbeiten in die Literaturübersicht integriert. Ergebnisse: Klare Zusammenhänge finden sich in der Literatur zwischen der Eltern-Kind-Beziehung und IBS bei Jugendlichen und jungen Erwachsenen. Die Qualität der Eltern-Kind-Kommunikation erscheint ein vielversprechender Ansatz zu sein, um eine IBS-Symptomatik zu beeinflussen. Es gibt Hinweise, dass zumindest für manche Familien die Förderung der gemeinsam verbrachten Zeit hilfreich sein könnte. In Bezug auf erzieherische Aspekte sollte zwischen verschiedenen Bereichen unterschieden werden. Elterliches Wissen und Kontrolle der Internetnutzung scheinen Schutzfaktoren zu sein, der Effekt von Regeln und Restriktionen ist jedoch ungeklärt. Schlussfolgerungen: Um eine IBS-Symptomatik zu verändern, ist es sinnvoll, die Eltern-Kind-Beziehung zu verbessern. Die Wege dorthin sind jedoch kaum erforscht. Empfehlungen in Bezug auf Erziehungsstrategien (z. B. Regeln und Restriktionen) sollten sehr vorsichtig formuliert werden, da die Effekte noch unklar sind. Die Integration der Eltern in Prävention und Intervention wird von vielen Forscher_innen befürwortet, es gibt jedoch nur einzelne Studien, die Eltern im Rahmen von Interventionen berücksichtigen.

Schlüsselwörter: Internetbezogene Störung, Internetsucht, Jugendliche, Familienfaktoren, Eltern

\section{Parental factors in internet and computer game addiction in adolescence: An overview}

Abstract: Objective: Internet-related disorders (IRD) in adolescents and young adults are closely linked to family factors. However, few research and review articles include the family. To this end, $N=87$ scientific papers were integrated into the literature review. Method: The present work gives an overview of parental factors that can be involved in the development, maintenance, and reduction of symptoms. Results: The literature provides clear connections between the parent-child relationship and IRD in adolescents and young adults. The quality of parent-child communication appears to be a promising approach for influencing IRD symptoms. There are indications that, at least for some families, encouraging the time spent together could be helpful. Regarding educational aspects, a distinction should be made between different areas. Parental knowledge and control of internet use seem to be protective factors. The effect of rules and restrictions, however, is unclear. Conclusions: To change IRD symptoms, it makes sense to improve the parent-child relationship. However, the ways to achieve that have hardly been explored. Recommendations regarding parenting strategies (e.g., rules and restrictions) should be formulated very carefully, as the effects are still unclear. The integration of parents in prevention and intervention efforts is advocated by many researchers, but there only individual studies have considered parents in the context of interventions.

Keywords: internet-related disorder, internet addiction, adolescent, family factors, parents

\section{Einleitung}

Internet- und Computerspielsucht betreffen in der Regel nicht nur die daran erkrankten Kinder, Jugendlichen oder jungen Erwachsenen, auch für ihre Familien und Angehörigen werden deren Auswirkungen spürbar. Dabei sind Eltern häufig an der Entwicklung, Aufrechterhaltung und dem Verlauf der Symptomatik beteiligt und leiden ebenso wie die Betroffenen darunter (Bonnaire, Liddle, Har, Niel- sen \& Phan, 2019; Bonnaire \& Phan, 2017; Lam \& Wong, 2015). Auch die Diagnosekriterien einer „Internet Gaming Disorder" nach DSM-5 (Diagnostic and Statistical Manual of Mental Disorders) weisen auf die enge Verknüpfung zu Familienfaktoren hin. So werden psychosoziale Auswirkungen, wie die Gefährdung oder der Verlust von wichtigen Familienbeziehungen oder das Täuschen von Familienmitgliedern, als Kriterien benannt (American Psychiatric Association, 2013). 
In der aktuellen Literatur zu Ursachen und Folgen einer Internet- oder Computerspielsucht finden sich hauptsächlich Übersichtsarbeiten zu individuellen Faktoren (z.B. männliches Geschlecht, jüngeres Alter, Angststörungen, Depression, Schlafprobleme, schlechte Schulleistungen, internetbezogene Motive und Nutzungsverhalten, kognitive Faktoren, Persönlichkeitsfaktoren; Anderson, Steen \& Stavropoulos, 2017; Cerniglia et al., 2017; Fumero, Marrero, Voltes \& Peñate, 2018; Lam, 2014; Männikkö, Ruotsalainen, Miettunen, Pontes \& Kääriäinen, 2020; Mihara \& Higuchi, 2017; Sugaya, Shirasaka, Takahashi \& Kanda, 2019). Nur wenige Übersichtsarbeiten adressieren Familien- und Kontextfaktoren (Schneider, King \& Delfabbro, 2017; Wartberg, Aden, Thomsen \& Thomasius, 2015). Welche Rolle aber spielen Eltern in der Entwicklung, Aufrechterhaltung und dem Verlauf dieser Symptomatik? Der vorliegende Artikel fasst aktuelle Literatur zu dieser Frage zusammen.

\section{Methodik}

Es wurde eine Literaturrecherche in deutscher und englischer Sprache durchgeführt. Dabei wurde Literatur berücksichtigt, die sich auf „Internetsucht“, „pathologische“, „problematische“, ,zwanghafte“ oder ,suchtartige“ Internetnutzung bezieht. Für die Darstellungen dieser Befunde wird der Begriff "Internetbezogene Störung“ (IBS) verwendet, auf den sich die Deutsche Gesellschaft für Suchtforschung und Suchttherapie e.V. (DG-Sucht) geeinigt hat (Rumpf et al., 2016). Da für die Computerspielsucht (CS) eigene Diagnosekriterien vorliegen und sich viele Studien explizit auf diesen Teilbereich beziehen, werden Befunde zur CS bzw. zur „problematischen“, „exzessiven“, „,suchtartigen“ Computerspielnutzung oder zur „Internet Gaming Disorder“ separat dargestellt. Andere Teilbereiche, die ebenfalls zu einer IBS gehören können (z. B. Sucht nach sozialen Netzwerken, Online-Pornografiesucht, Online-Glücksspielsucht, Online-Kaufsucht), werden in dieser Literaturübersicht nicht behandelt. Sofern lediglich eine gesteigerte Internetnutzungszeit und nicht eine pathologische Nutzung berichtet wurde, wurde dies entsprechend beschrieben. Medienerzieherische Aspekte wurden nur im Zusammenhang mit der Prävention einer IBS berücksichtigt. Es wurden nur Artikel einbezogen, die Kinder, Jugendliche und junge Erwachsene bzw. deren Eltern untersuchen.

\section{Ergebnisse}

Es konnten $N=87$ Studien identifiziert werden, deren Ergebnisse in vier Kategorien eingeteilt wurden. Die erste Kategorie behandelt die Aspekte der Eltern-Kind-Beziehung (inkl. gemeinsam verbrachte Zeit, Eltern-Kind-Kommunikation) sowie den Aspekt der Familienfunktionalität. Die zweite Kategorie betrachtet das elterliche Erziehungsverhalten (elterliches Wissen und Kontrolle, Regeln und Restriktion, elterliche Einstellung und Vorbildfunktion). Die dritte Kategorie adressiert Belastungsfaktoren, die in Familien vorliegen können. Die vierte Kategorie behandelt familiäre Kontextfaktoren (z.B. sozioökonomischer Status). Die Studienlage zu IBS und CS wird im Folgenden anhand dieser auch für klinische Interventionen relevanten Kategorien dargestellt.

\section{Einfluss der Eltern-Kind-Beziehung und Familienfunktionalität}

Die meisten Studienergebnisse zu Elternfaktoren im Zusammenhang mit IBS beziehen sich auf die Eltern-KindBeziehung. Studien fanden bei einfacher Betrachtung einen direkten Zusammenhang zwischen einer guten Eltern-Kind-Beziehung und einer geringen Internetnutzung (Mesch, 2009; Richards, McGee, Williams, Welch \& Hancox, 2010; Sanders, Field, Diego \& Kaplan, 2000). Weitere Studien brachten weniger Symptome einer IBS (King \& Delfabbro, 2017; C.-Y. Liu \& Kuo, 2007; Tsitsika et al., 2011; W. Wang et al., 2018; X. Wu et al., 2013) oder CS (Hwang, Hong, Kim \& Han, 2020; Kim \& Kim, 2015; C.W. Wang et al., 2014; Zorbas, Ulas \& Kizildag, 2015) in Zusammenhang mit der Eltern-Kind-Beziehung. In einzelnen Studien wurden Hinweise auf mögliche Mediatoren, wie die Nutzung des Internets für Lernzwecke (Mesch, 2009), soziale Ängstlichkeit (C.-Y. Liu \& Kuo, 2007), Sozialkontakte (C.-Y. Liu \& Kuo, 2007; Zhu, Zhang, Yu \& Bao, 2015), Schulverbundenheit (Zhu et al., 2015), Emotionsregulationsfähigkeit (W. Wang et al., 2018) oder elterliche Paarprobleme berichtet (Yang, Zhu, Chen, Song \& Wang, 2016). Venkatesh, Sykes, Chan, Thong und Hu (2019) zeigten, dass die Eltern-Kind-Beziehung aus Sicht des Kindes ein wesentlicher Moderator ist zwischen elterlichem Erziehungsverhalten und einer IBS-Symptomatik. Mehrere Studien belegen, dass Jugendliche mit IBS aus ihrer Sicht weniger elterliche Fürsorge und emotionale Wärme erfahren (Faltýnková, Blinka, Ševčíková \& Husarova, 2020; Karaer \& Akdemir, 2019; Kwon, Chung \& Lee, 2011; Li, Li \& Newman, 2013; Y.-H. Lin \& Gau, 2013; Siomos et al., 2012; Xiuquin et al., 2010) und stattdessen Kontrolle und Überprotektion wahrnehmen (Faltýnková et al., 2020; Siomos et al., 2012; Xin et al., 2018; Xiuquin et al., 2010). 
Längsschnittstudien zeigen, dass die Eltern-Kind-Beziehung sowohl in der Entstehung einer IBS Bedeutung besitzt (Choo, Sim, Liau, Gentile \& Khoo, 2015; Liau et al., 2015; Shek, Zhu \& Dou, 2019; Zhu et al., 2015) als auch in Folge des Suchtverhaltens beeinträchtigt werden kann (Da Charlie, HyeKyung \& Khoo, 2011; Gentile et al., 2011). Im Längsschnitt zeigte sich auch, dass fehlende elterliche Warmherzigkeit und Fürsorge mit späteren IBS-Symptomen assoziiert ist (Chen, Chen \& Gau, 2015; Liau et al., 2015; Tóth-Király, Morin, Hietajärvi \& Salmela-Aro, 2021). Eine therapeutisch angeregte Verbesserung der ElternKind-Beziehung zeigte Effekte auf die IBS-Symptomatik von Jugendlichen (Q.-X. Liu et al., 2015).

Bezüglich der Unterschiede in der Ausprägung der Eltern-Kind-Beziehung bei Müttern oder Vätern ist die aktuelle Datenlage heterogen. Einzelne Studien deuten darauf hin, dass die Beziehung zu den Müttern in Bezug auf manche Aspekte relevanter ist (Da Charlie et al., 2011; Q.-X. Liu, Fang, Zhou, Zhang \& Deng, 2013; Tóth-Király et al., 2021; J. Xu et al., 2014), während andere Studien Aspekte der Beziehung zum Vater hervorheben (Da Charlie et al., 2011; Q.-X. Liu et al., 2013; Rehbein \& Baier, 2013; Su et al., 2018; Tóth-Király et al., 2021) oder beiden Beziehungen gleich viel Bedeutung einräumen (Shek et al., 2019).

Die bisherige Literatur weist zusammenfassend stark darauf hin, dass die Eltern-Kind-Beziehung ein wesentlicher Einflussfaktor bei der Entwicklung einer IBS sein kann. Im Rahmen der Prävention und Intervention bestehen Ansätze, die Eltern-Kind-Beziehung positiv zu beeinflussen, beispielsweise durch die aktive Förderung gemeinsamer positiver Zeit zwischen Eltern und Kind (siehe Han, Kim, Lee \& Renshaw, 2012) oder die Verbesserung der Eltern-Kind-Kommunikation (siehe Q.-X. Liu et al., 2015).

\section{Gemeinsame Zeit zwischen Eltern und Kind}

Zur gemeinsam verbrachten Zeit zwischen Eltern und Kind liegen im Kontext einer IBS nur einzelne Studien vor. Diese zeigen, dass je mehr Zeit die Eltern mit ihren Kindern verbringen, desto weniger IBS-Symptome liegen bei den Kindern vor (Faltýnková et al., 2020; Jeong \& Kim, 2011; C.-H. Lin, Lin \& Wu, 2009). Umgekehrt betrachtet weist eine querschnittliche Studie darauf hin, dass eine gesteigerte Internetnutzungszeit mit einer wahrgenommenen Reduktion der Familienzeit einhergeht (Lee \& Chae, 2007). Die Rolle der Eltern-Kind-Beziehung in Bezug auf die Familienzeit ist in diesem Zusammenhang jedoch nahezu unerforscht. Eine Studie beschrieb einen positiven Zusammenhang zwischen gemeinsam verbrachter Zeit und der Eltern-Kind-Beziehung, allerdings konnte dabei keine Verbindung zur Art und Dauer der Internetnutzung hergestellt werden (Mesch, 2009). Han et al. (2012) konnten zeigen, dass sich u.a. durch eine Steige- rung der Familienzeit die Bildschirmzeiten der Jugendlichen reduzieren lassen.

\section{Eltern-Kind-Kommunikation}

Bonnaire et al. (2019) beschrieben in ihrem Artikel, dass die Kommunikation in Familien mit Jugendlichen mit IBS häufig von Vorwürfen, Verteidigung und gegenseitigen Schuldzuweisungen geprägt ist, worauf sich manche Jugendliche weiter in die virtuelle Welt zurückzögen. Verschiedene Studien finden einen direkten Zusammenhang zwischen einer negativen Familienkommunikation und mehr Symptomen einer IBS (Faltýnková et al., 2020; Q.-X. Liu, Fang, Deng \& Zhang, 2012; Park, Kim \& Cho, 2008; Tsitsika et al., 2011; van den Eijnden, Spijkerman, Vermulst, van Rooij \& Engels, 2010). Weitere Studien finden einen positiven Zusammenhang zwischen IBS und Familienkonflikten (De Leo \& Wulfert, 2013; C.-F. Yen, Ko, Yen, Chang \& Cheng, 2009; J.-Y. Yen, Yen, Chen, Chen \& Ko, 2007), Ermahnen und Warnen vor Computerspielen (Z. Xu, Turel \& Yuan, 2012) oder dem Äußern von Kritik (De Leo \& Wulfert, 2013). Auch Längsschnittstudien weisen auf einen negativen Zusammenhang zwischen Familienkommunikation und späteren IBS-Symptomen hin (Yu \& Shek, 2013). Van den Eijnden et al. (2010) differenzierten in ihrer Studie nach Qualität und Quantität der Kommunikation und fanden, dass Defizite in der Qualität der Eltern-Kind-Kommunikation mit späteren IBS-Symptome assoziiert waren. IBS-Symptome beim Kind hängen außerdem im Verlauf mit einer Reduktion der Quantität der Kommunikation zusammen (siehe auch Kraut et al., 1998). Q.-X. Liu et al. (2015) konnten zeigen, dass sich die Familienkommunikation durch ein Gruppentraining für Eltern und Jugendliche verbessern lässt und dass diese Veränderungen mit einer Reduktion einer IBS-Symptomatik einhergeht.

\section{Familienfunktionalität und andere Konstrukte}

Unter dem Begriff Familienfunktionalität werden verschiedene Aspekte von Familienbeziehungen, Kommunikation und Familienklima subsummiert. Der Begriff wird in Studien jedoch höchst unterschiedlich operationalisiert, was eine zusammenfassende Interpretation der Literatur erschwert. J.-Y. Yen et al. (2007) definierten Familienfunktionalität beispielsweise durch erlebte familiäre Hilfe und Unterstützung, Emotionalität und gemeinsame Zeit. Chiu, Lee und Huang (2004) beschreiben Familienfunktionalität als subjektiv erlebte Harmonie und Interaktion in der Familie. Aufgrund der Vielzahl der Studien soll das Konstrukt der Familienfunktionalität trotz der heterogenen Definition hier berücksichtigt werden.

Während verschiedene Querschnitterhebungen einen negativen Zusammenhang zwischen Familienfunktionalität und Symptomen einer IBS (Cacioppo et al., 2019; 
Kammerl et al., 2012; Rikkers, Lawrence, Hafekost \& Zubrick, 2016; Shi, Wang \& Zou, 2017; J.-Y. Yen et al., 2007) und CS (Chiu et al., 2004; Rikkers et al., 2016; Wartberg, Zieglmeier \& Kammerl, 2019) herstellen, kommen Längsschnittdaten zu heterogenen Ergebnissen. Manche Studien konnten dabei eine geringe Familienfunktionalität als relevanten Prädiktor bei der Entwicklung einer IBS identifizieren (Ko, Yen, Yen, Lin \& Yang, 2007; Yu \& Shek, 2013), andere hingegen nicht (Chen et al., 2015; Shek \& Yu, 2016). Einzelne Studien zeigten, dass der Zusammenhang zwischen Familienfunktionalität und IBS mediiert wird durch die Emotionsregulationskompetenz der Jugendlichen (Zhang, Luo, Yao \& Barrow, 2021) oder einem geringen Selbstwert oder Einsamkeitsgefühlen (Shi et al., 2017). Cacioppo et al. (2019) fanden nur für manche Aspekte der Familienfunktionalität negative $\mathrm{Zu}-$ sammenhänge mit IBS (Problemlöseverhalten in der Familie, Rollenverhalten, affektive Ansprechbarkeit, affektives Involviertsein), während andere Teilbereiche keinen signifikanten Zusammenhang zeigten (Kommunikation, Verhaltenskontrolle).

In der Literatur finden sich außerdem einzelne Studien zu weiteren Konstrukten, die Familienfaktoren beschreiben. Diese zeigen z.B. einen negativen Zusammenhang zwischen der subjektiven Familienunterstützung und der Internetnutzungsdauer (Appel, Holtz, Stiglbauer \& Batinic, 2012) oder IBS-Symptomen (Ergün \& Işık, 2018). Andere Studien finden Zusammenhänge zwischen einer erlebten Einsamkeit in der Familie und IBS (İskender, 2018), Familienkohäsion und IBS (Chung, Lee \& Lee, 2019; Park et al., 2008) oder zwischen Familiendysharmonie und IBS (Tsitsika et al., 2011) und CS (C.-W. Wang et al., 2014). Längsschnittdaten weisen darauf hin, dass eine hohe Familienkohäsion den Einfluss von sozialer Ängstlichkeit auf eine CS-Symptomatik reduzieren kann (Adams et al., 2019).

Zusammenfassend lässt sich sagen, dass die meisten Studien einen Zusammenhang herstellen zwischen dem Funktionsniveau einer Familie und IBS-Symptomen beim Kind. Die Ursache-Wirkungs-Beziehung ist jedoch noch nicht geklärt.

\section{Einfluss der Erziehung}

Die Forschung zu erzieherischen Aspekten im Rahmen von IBS differenziert zunehmend danach, was Eltern woher wissen und $o b$ und wie sie die Internetnutzung ihrer Kinder reglementieren oder einschränken. Im Folgenden wird daher unterschieden nach elterlichem Wissen und der Quelle der Information (Kontrolle), nach dem Einsatz von Regeln und Restriktionen sowie nach der elterlichen Einstellung zum Internet und der Vorbildfunktion.

\section{Elterliches Wissen und Kontrolle}

Einzelne Forschungsarbeiten im Kontext von IBS zeigen, dass ein hohes Maß an elterlichem Wissen z.B. über den Aufenthaltsort oder die Aktivitäten des Kindes (Durkee et al., 2012; Faltýnková et al., 2020) oder über die Internetnutzung des Kindes mit weniger CS-Symptomen (C.-H. Lin et al., 2009) bzw. mit einer geringeren Internetnutzungsdauer einhergeht (Valcke, Bonte, Wever \& Rots, 2010). Eine qualitative Studie diskutierte hingegen, dass sich Jugendliche von elterlicher Kontrolle bedrängt fühlen könnten, was im gegenteiligen Effekt zur Flucht in die virtuelle Welt führen könnte (Siste et al., 2020). Dies wurde auch in einer Querschnitterhebung bestätigt, die einen negativen Zusammenhang zwischen elterlicher Kontrolle und IBS-Symptomen beobachten konnte (van Petegem, Ferrerre, Soenens, van Rooij \& van Looy, 2019).

Verschiedene andere Studien zeigen jedoch, dass elterliche Kontrolle, oft als elterliches „Monitoring“ bezeichnet (vgl. Kammerl \& Wartberg, 2018), mit geringeren Internetnutzungszeiten (Valcke et al., 2010) oder weniger Symptomen einer IBS einhergeht (Bonnaire \& Phan, 2017; Oh, 2003; Siomos et al., 2012; Z. Xu et al., 2012). Außerdem weist eine Studie darauf hin, dass der Einfluss von elterlicher Kontrolle (Webseiten prüfen, Profile und Kontakte verfolgen, Nachrichten lesen, Kontrollsoftware verwenden) oder aktiver Beteiligung der Eltern (miteinander sprechen, anleiten, Hilfestellung geben, gemeinsam nutzen) eher gering zu sein scheint (Kalmus, Blinka \& Olafsson, 2015).

Ob Eltern aktiv kontrollieren oder nicht, hängt außerdem von verschiedenen Faktoren ab. Weniger Kontrolle wird von den Eltern z.B. durchgeführt, wenn die Jugendlichen eine hohe Selbstregulationskompetenz zeigen (Padilla-Walker \& Coyne, 2011), die Kinder älter sind und die Eltern ihre eigene Kompetenz mit dem Internet als gering einschätzen (Valcke et al., 2010). Mehr Kontrolle wird ausgeübt, wenn die Eltern eine negative Einstellung gegenüber Computerspielen aufweisen (van Petegem et al., 2019). Mütter scheinen außerdem mehr Kontrolle auszuüben als Väter (Valcke et al., 2010).

\section{Regeln und Restriktion}

Äußerst heterogen stellt sich die Datenlage in Bezug auf Regelung und Restriktion der Internetnutzung der Kinder durch die Eltern dar. Manche Querschnitterhebungen finden einen negativen Zusammenhang zwischen aufgestellten Regeln und Symptomen einer IBS (Bonnaire \& Phan, 2017; Chng, Li, Liau \& Khoo, 2014; Li et al., 2013; Martins et al., 2020; Siomos et al., 2012; van Petegem et al., 2019). Chng et al. (2014) fanden, dass diese Assoziation stärker war in Familien mit besserer Eltern-Kind-Beziehung und stärkerer Bindung. Auch Längsschnittdaten bestätigen diesen Zusammenhang (Rehbein \& Baier, 2013). Man 
könnte also schlussfolgern, dass Regelungen und Restriktionen einen protektiven Wert haben, der sich in funktionierenden Familien stärker äußert.

Gleichzeitig gibt es Querschnitterhebungen, die einen positiven Zusammenhang herstellen. Demnach steht elterliche Restriktion in Verbindung mit mehr Symptomen einer IBS (Kalmus et al., 2015; C.S.T. Wu et al., 2016) oder CS (Z. Xu et al., 2012). Die Restriktion von Ressourcen (z.B. Taschengeld, Verfügbarkeit von Computer) stand in Zusammenhang mit mehr CS-Symptomen (Z. Xu et al., 2012). Eine Interpretation dieser Ergebnisse ist aufgrund des Studiendesigns nicht eindeutig möglich, so könnte Restriktion zu mehr Spiellust führen oder Restriktionen von den Eltern als Reaktion auf problematisches Verhalten eingesetzt werden.

Ein reziproker Zusammenhang zeigt sich in den Längsschnittdaten von Su et al. (2018). Mehr Kontrolle und Restriktion waren negativ mit einer CS-Symptomatik 6 Monate später assoziiert. Außerdem zeigte sich im weiteren Verlauf, dass CS-Symptome zu weniger Kontrolle und Restriktion führten.

Des Weiteren gibt es Querschnitt- (Kammerl \& Wartberg, 2018) und Längsschnittstudien (Choo et al., 2015; Liau et al., 2015), die keinen Zusammenhang zwischen Restriktion und IBS-Symptomen beobachten konnten.

Manche Studien finden unterschiedliche Ergebnisse, wenn man nach inhaltlicher oder zeitlicher Begrenzung differenziert (van den Eijnden et al., 2010). Eine Restriktion der Nutzungsdauer stand dabei in Zusammenhang mit mehr IBS-Symptomen, eine inhaltliche Begrenzung mit weniger IBS-Symptomen. Andere Forscher_innen fanden auch unter dieser Differenzierung keinen Zusammenhang zu einer CS-Symptomatik (Lee \& Chae, 2007).

Hinzu kommen Befunde, dass inkonsistente Regelungen schädlich zu sein scheinen (Kammerl \& Wartberg, 2018; Kveton \& Jelinek, 2016; Xin et al., 2018) ebenso wie rigide und chaotische Regeln (im Vergleich zu flexiblen und strukturierten Regelungen; Tafà \& Baiocco, 2009; Valcke et al., 2010). Fidan und Seferoglu (2020) diskutieren in ihrem Review, dass Eltern einen restriktiven Ansatz wählen, wenn sie ihre Kompetenz mit digitalen Technologien als gering einschätzen (siehe auch Siomos et al., 2012). Der Einsatz von Regeln erfordere kaum inhaltliche Kompetenz und erscheine am einfachsten.

\section{Elterliche Einstellungen und Vorbildfunktion}

Während einzelne Studien berichten, dass Eltern, die der Internetnutzung ihrer Kinder ablehnend gegenüberstehen (Jeong \& Kim, 2011; J. Xu et al., 2014) oder das Internet gar nicht nutzen (Kalmus et al., 2015), häufiger Kinder haben, die eine IBS-Symptomatik aufweisen, kommen andere Studien zu entgegengesetzten Schlüssen. So zeigen einzelne Studien einen positiven Zusammenhang zwischen der elterlichen Einstellung zum Internet und der Nutzungsdauer beim Kind (Valcke et al., 2010) oder zwischen einer grundsätzlich positiven Einstellung zu Suchtmitteln und IBS-Symptomen (J.-Y. Yen et al., 2007). Q.-X. Liu et al. (2012) betrachteten die Zusammenhänge differenziert und fanden, dass das Elternverhalten mehr Relevanz hatte als die Einstellung der Eltern.

Auch andere Studien zeigen, dass die Häufigkeit elterlichen Computerspielens mit CS-Symptomen bei den Jugendlichen (J.Y.W. Wu, Ko, Wong, Wu \& Oei, 2016) und das Ausmaß der elterlichen Internetnutzung mit dem der Internetnutzung von Jugendlichen einhergeht (Valcke et al., 2010). Zwei Untersuchungen fanden außerdem positive Assoziationen zwischen einer IBS-Symptomatik bei den Eltern und beim Kind (Lam, 2020; Lam \& Wong, 2015). Jugendliche zeigten in diesem Fall ein dreifach erhöhtes Risiko, selbst eine IBS-Symptomatik zu entwickeln (Lam \& Wong, 2015).

\section{Familiäre Belastungsfaktoren und Erkrankung der Eltern}

Verschiedene Studien bringen Symptome einer IBS in Zusammenhang mit Belastungen im familiären Kontext. So zeigen Studien positive Zusammenhänge zwischen IBS und einer ablehnenden Haltung der Eltern gegenüber der oder dem Jugendlichen (z.B. „Meine Eltern mögen mein Verhalten nicht, egal, was ich tue.“; Kim \& Kim, 2015; Kwon et al., 2011; Xiuquin et al., 2010), emotionalem oder physischem Missbrauch (Schimmenti, Passanisi, Gervasi, Manzella \& Famà, 2014), strafendem Verhalten durch die Eltern (Xiuquin et al., 2010), Konflikte zwischen den Eltern (Xin et al., 2018; J.-Y. Yen et al., 2007; Zhang, Spinrad, Eisenberg, Luo \& Wang, 2017) oder Gewalt zwischen den Eltern oder gegenüber der oder dem Jugendlichen (Park et al., 2008; Vadlin, Åslund, Hellström \& Nilsson, 2016). Des Weiteren zeigen Studien, dass Symptome einer IBS bei den Jugendlichen in Zusammenhang stehen mit psychischen Erkrankungen der Eltern, z.B. Depression, Angststörungen, Alkoholprobleme (Jang \& Ji, 2012; Lam, 2015; Rikkers et al., 2016; Wartberg et al., 2017) oder einer IBSSymptomatik (s. o.).

\section{Familiäre Kontextfaktoren}

In Bezug auf Kontextfaktoren gibt es eine starke Heterogenität zwischen den Studien. Manche Untersuchungen finden negative Zusammenhänge zwischen dem Familieneinkommen oder dem sozioökonomischen Status und IBS (Shek et al., 2019; C.S. T. Wu et al., 2016), andere nicht (Q.-X. Liu et al., 2012; Park et al., 2008; C.-W. Wang et al., 
2014; J. Xu et al., 2014; J.-Y. Yen et al., 2007). Einzelne Studien berichten von einem positiven Zusammenhang dieser Aspekte mit IBS (X.-S. Wu et al., 2016; Yu \& Shek, 2013). Auch hinsichtlich eines Zusammenhangs zwischen IBS und dem Bildungsstand der Eltern gibt es keine eindeutigen Daten (Q.-X. Liu et al., 2012; Tsitsika et al., 2011; C.-W. Wang et al., 2014). Während manche Studien berichten, dass Eheprobleme der Eltern (Zhang et al., 2017) oder eine Scheidung oder Trennung der Eltern (Tsitsika et al., 2011; C.S.T. Wu et al., 2016) einflussreich sind, findet sich dieser Befund nicht in anderen Erhebungen (Shek \& Yu, 2016; C.-W. Wang et al., 2014; J.-Y. Yen et al., 2007; Yu \& Shek, 2013). Kinder, die nur bei einem Elternteil aufwachsen, zeigten in einer Studie ein erhöhtes Risiko, eine CS-Symptomatik zu entwickeln (Rehbein \& Baier, 2013). Kein Einfluss scheint es zu haben, ob ein Kind Einzelkind ist oder mit Geschwistern aufwächst (Durkee et al., 2012; C.-W. Wang et al., 2014; C.S. T. Wu et al., 2016) und ob die Familie Migrationserfahrung hat oder nicht (Durkee et al., 2012; Yu \& Shek, 2013).

\section{Diskussion}

Die vorliegende Arbeit gibt einen Überblick über die wissenschaftliche Literatur zu Elternfaktoren bei IBS und CS bei Jugendlichen und jungen Erwachsenen. Es konnten klare Zusammenhänge festgestellt werden sowohl zwischen IBS und der Eltern-Kind-Beziehung als auch spezifisch zwischen CS und der Eltern-Kind-Beziehung (z.B. Hwang et al., 2020; W. Wang et al., 2018). Längsschnittstudien weisen darauf hin, dass die Eltern-Kind-Beziehung als relevanter Prädiktor zu betrachten ist (z.B. Shek et al., 2019), aber auch in Folge des Suchtverhaltens beeinträchtigt werden kann (z.B. Da Charlie et al., 2011). Daraus kann abgeleitet werden, dass die Eltern-Kind-Beziehung sowohl in der Prävention als auch in der Behandlung einer IBS- oder CS-Symptomatik berücksichtigt werden sollte. Nur einzelne Studien erforschen mögliche Mediatoreffekte, zwei davon weisen auf die soziale Kompetenz der Jugendlichen als relevanter Mediator hin (soziale Ängstlichkeit, Sozialkontakte, Verbundenheit zu Lehrer innen und Mitschüler_innen; C.-Y. Liu \& Kuo, 2007; Zhu et al., 2015). Daraus könnte man ableiten, dass ein soziales Kompetenztraining bei Jugendlichen helfen könnte, negative Einflüsse durch die Eltern-Kind-Beziehung zu kompensieren. Studien hierzu fehlen allerdings noch.

Für die klinische Praxis stellt sich die Frage, wie die Eltern-Kind-Beziehung im Kontext von IBS adressiert werden kann. Naheliegend ist, die Familien zu gemeinsamer positiver Zeit zu motivieren. Es gibt jedoch nur einzelne Studien, die darauf hinweisen, dass mehr gemeinsame
Zeit mit weniger IBS-Symptomen assoziiert ist, deren Ursache-Wirkungs-Beziehung unklar ist (z.B. Faltýnková et al., 2020). Eine Interventionsstudie konnte u.a. durch eine Steigerung der gemeinsamen Familienzeit eine Reduktion der Bildschirmzeit erreichen (Han et al., 2012). Besonders im Jugendalter, in dem die Autonomieentwicklung zentral ist und Peer-Kontakte den Familienkontakten vorgezogen werden, ist diese Herangehensweise vermutlich jedoch oft schwierig. In dem Zusammenhang zeigen mehrere Forschungsarbeiten, dass Eltern von Kindern mit IBS vermehrt überprotektives Verhalten zeigen (z. B. Einmischung, exzessiver Kontakt, Kontrolle, Verhinderung der Entwicklung zu Unabhängigkeit und Autonomie; Siomos et al., 2012). Schlussfolgernd liegt die Vermutung nahe, dass gemeinsame positive Zeit zwischen Eltern und Kind, in der Eltern und Kind näher zusammenrücken, für manche Familien hilfreich sein kann und in anderen Familien eher die Förderung der Autonomieentwicklung der Jugendlichen, in der Eltern die Kinder ziehen lassen, sinnvoll ist. Forschungsdaten, welche diese Prozesse und die individuellen Faktoren einer Familie näher beleuchten, sind wünschenswert.

Ein weiterer Faktor, der auf die Eltern-Kind-Beziehung wirken könnte, ist die Eltern-Kind-Kommunikation. Mehrere Forschungsarbeiten weisen auf den Zusammenhang zwischen Eltern-Kind-Kommunikation und IBS hin und schildern Konflikte, die häufig zwischen Eltern und Kindern im Zusammenhang mit der Internetnutzung auftreten (z. B. Faltýnková et al., 2020). Die Sorge der Eltern vor Gefahren im und mit dem Internet und deren Wunsch nach Kontrolle und Sicherheit trifft dabei auf das Unverständnis der Jugendlichen und deren Wunsch nach Vertrauen, Autonomie und Privatsphäre (Borca, Bina, Keller, Gilbert \& Begotti, 2015). Eine Längsschnittstudie zeigte, dass eine qualitativ hochwertige Kommunikation (z.B. sich verstanden, ernst genommen und wohl in der Kommunikation mit den Eltern über das Internet fühlen) in Zusammenhang steht mit weniger IBS-Symptomen beim Kind, während eine IBS-Symptomatik mit einer reduzierten Häufigkeit der Kommunikation einhergeht (van den Eijnden et al., 2010). Auch wenn diese Ergebnisse repliziert werden müssen und nur als vorläufig betrachtet werden können, erscheint die Berücksichtigung der Qualität der Eltern-Kind-Kommunikation im Rahmen der Therapie einer IBS erfolgversprechend (siehe auch Han et al., 2012).

Erziehungsratgeber, die Eltern im Umgang mit der Medienerziehung beraten, beziehen sich häufig auf Hinweise zu Kontrolle, Regeln und Restriktionen. Ca. 48\% der Eltern in Deutschland reglementieren die Dauer der Internetnutzung ihrer Jugendlichen, $44 \%$ auch den Inhalt (Medienpädagogischer Forschungsverbund Südwest, 2017). Die wissenschaftliche Literatur zeichnet jedoch kein ein- 
heitliches Bild zum Einfluss dieser Aspekte auf die Entwicklung einer IBS. Es gibt ebenso viele Studien, die einen negativen Zusammenhang zwischen Regeln und Restriktion und IBS-Symptomen herstellen, wie auch Studien, die einen positiven oder auch keinen Zusammenhang herstellen, sowohl im Querschnitt als auch im Längsschnitt (z.B. Choo et al., 2015; Martins et al., 2020; C.S.T. Wu et al., 2016). Su et al. (2018) berichteten in ihrer Studie außerdem von einem wechselseitigen Prozess zwischen Regeln und Restriktionen und einer IBS-Symptomatik. Dies deckt sich mit der klinischen Erfahrung, wonach Regelungen, die von Eltern oft einmalig ausgesprochen, aber inkonsequent und chaotisch umgesetzt wurden (siehe auch Kammerl \& Wartberg, 2018; Xin et al., 2018), dann verschärft werden, wenn das Kind erste Symptome einer IBS entwickelt. Ratgeber, die den Einsatz von Regeln und Restriktionen zur Prävention einer IBS-Symptomatik empfehlen, sollten ihre Ratschläge mit Vorsicht formulieren und eine flexible, aber dennoch konsequente und strukturierte Umsetzung der Regelungen empfehlen. Zukünftige Forschung sollte mehr Licht ins Dunkel bringen und differenziert erheben (z. B. Reglementierung von Inhalt, Zeitpunkt, Dauer; vgl. Van den Eijnden et al., 2010), die Umsetzung der Regeln berücksichtigen (z. B. Konsequenz vs. Inkonsequenz; vgl. Kveton \& Jelinek, 2016) sowie den Einfluss persönlicher Faktoren des Kindes (z. B. Selbstregulationskompetenz; vgl. Padilla-Walker \& Coyne, 2011), der Eltern (z.B. digitale Kompetenz; vgl. Fidan \& Seferoglu, 2020) und der Eltern-Kind-Beziehung (siehe Chng et al., 2014) erfassen.

In Bezug auf die elterliche Kontrolle zeigen die meisten Studien, dass mehr Kontrolle mit weniger IBS-Symptomen einhergeht, auch wenn der Einfluss begrenzt zu sein scheint (z.B. Bonnaire \& Phan, 2017; Kalmus et al., 2015). Dabei wird elterliches Wissen z.B. über den Aufenthaltsort des Kindes oder auch die Internetnutzung des Kindes als Schutzfaktor betrachtet (z.B. Faltýnková et al., 2020; C.-H. Lin et al., 2009). Kerr, Stattin und Burk (2010) beschreiben in ihren Forschungsarbeiten jedoch, dass elterliches Wissen grundsätzlich nur dann einen protektiven Wert hat, wenn es auf der freiwilligen Selbstkundgabe der Jugendlichen basiert. Durch Kontrolle erzwungene Information ist gemäß den Autor_innen grundsätzlich schädlich. Dies deckt sich mit den Ergebnissen einzelner Forschungsarbeiten im Kontext von IBS, die einen positiven Effekt von Regeln und einen negativen Effekt von Kontrolle beobachten konnten (van Petegem et al., 2019). Im größten Teil der Forschungsliteratur zu IBS finden sich jedoch kaum Studien, welche die Aspekte Wissen und Kontrolle voneinander trennen. Gerade bei der Nutzung mobiler Endgeräte, die sich der Kontrolle der Eltern weitgehend entzieht, wäre eine solche Differenzierung aber wünschenswert. Für die Praxis könnte man aus diesen Erkenntnissen ableiten, ob man Eltern zur Kontrolle motivieren oder ihnen eher raten soll, mit ihren Kindern in einem vertrauensvollen Dialog zu sein, ohne zu kontrollieren.

Bei der elterlichen Einstellung zum Internet lässt sich ein U-förmiger Zusammenhang vermuten. Eine stark negative Einstellung scheint in einzelnen Studien ebenso in Zusammenhang zu IBS beim Kind zu stehen wie eine sehr positive Einstellung (z.B. Valcke et al., 2010; J. Xu et al., 2014). Einzelne Forschungsdaten weisen darauf hin, dass die Vorbildfunktion relevanter zu sein scheint als die Einstellung zum Internet (Q.-X. Liu et al., 2012). Die klinische Erfahrung zeigt, dass sich zwischen Eltern und Kind oft Fronten bilden, die in permanenter Angriffs- und Abwehrhaltung gegenüberstehen. Ein Aufweichen dieser beiden extremen Einstellungen ist im therapeutischen Prozess oft ein erster Schritt, um wieder miteinander in einen Dialog auf Augenhöhe zu gelangen.

Nur wenige Forschungsarbeiten berücksichtigen den Einfluss einer psychischen Erkrankung der Eltern auf die Entwicklung einer IBS beim Kind. In diesen wurden $\mathrm{Zu}-$ sammenhänge zu Angst und Depression, Alkoholproblemen und einer eigenen IBS-Symptomatik gefunden (Jang \& Ji, 2012; Lam, 2015; Rikkers et al., 2016; Wartberg et al., 2017). Auch hier ist wiederum unklar, wie der kausale Zusammenhang zu bewerten ist. Psychische Erkrankungen der Eltern könnten für Jugendliche belastend sein, die daraufhin das Internet als "sicheres Refugium“ nutzen und in eine IBS- oder CS-Symptomatik rutschen (vgl. Lam, 2020). Ebenso könnten IBS- oder CS-Symptome bei Jugendlichen zu starken Belastungen bei den Eltern führen, die wiederum in psychischen Symptomen münden könnten. Bonnaire et al. (2019) schilderten dazu in ihrem Artikel, dass sich Eltern von Jugendlichen mit CS entmutigt, erschöpft und überfordert fühlen und Schuldgefühle sowie einen reduzierten elterlichen Selbstwert entwickeln können. In der klinischen Praxis sollte eine mögliche Symptomatik der Eltern berücksichtigt werden, v.a. im Hinblick auf Ängste, Depressionen oder Sucht, um entsprechende Hilfsangebote machen oder verweisen zu können. Eltern sollten in ihrem Selbstwert und ihrer Selbstwirksamkeitserwartung gestärkt werden, um eine Therapie des Jugendlichen oder jungen Erwachsenen unterstützen zu können. Außerdem zeigen die Ergebnisse, dass Eltern eine Anlaufstelle zur Beratung im Umgang mit einer IBSSymptomatik ihres Kindes brauchen, auch wenn ihrem Kind die Problemeinsicht fehlt und es sich nicht zu einer Behandlung motivieren lässt (siehe auch Bonnaire et al., 2019; Zajac, Ginley, Chang \& Petry, 2017).

Im Einklang damit kommen sämtliche Studien, die Familienfaktoren in Zusammenhang mit IBS oder CS untersuchten, zu dem Schluss, dass die Integration von Eltern in die Behandlung sinnvoll ist (z. B. Bonnaire et al., 2019; Ca- 
cioppo et al., 2019; Han et al., 2012; Karaer \& Akdemir, 2019; Liau et al., 2015; C.-Y. Liu \& Kuo, 2007; Schneider et al., 2017; Throuvala, Janikian, Griffiths, Rennoldson \& Kuss, 2019) bzw., dass die Kombination von intrapersonalen und interpersonellen Strategien die besten Erfolge ermöglicht (Bussone, Trentini, Tambelli \& Carola, 2020). Vor allem die Förderung einer positiven Eltern-Kind-Beziehung (Karaer \& Akdemir, 2019; Kim \& Kim, 2015; C.-Y. Liu \& Kuo, 2007; Yu \& Shek, 2013), v.a. zu den Vätern (Schneider et al., 2017; Throuvala et al., 2019), sowie eine Veränderung von dysfunktionalen Kommunikationsmustern (Bonnaire et al., 2019; Yu \& Shek, 2013) stehen bei den Empfehlungen im Fokus. Dennoch adressieren sowohl Präventionsprogramme (Szasz-Janocha, Kindt, Halasy \& Lindenberg, 2019; Vondráčková \& Gabrhelík, 2016) als auch Interventionsprogramme fast ausschließlich die Betroffenen (Cerniglia et al., 2017; Stevens, King, Dorstyn \& Delfabbro, 2019; Zajac et al., 2017).

\section{Beteiligung von Eltern an Prävention und Intervention} In Deutschland werden die Eltern hauptsächlich im Rahmen der Prävention und Frühintervention angesprochen, um im frühen Stadium die Entwicklung einer voll ausgeprägten IBS-Symptomatik beim Kind zu verhindern. Hierfür stehen beispielsweise onlinebasierte Portale wie ,insnetz-gehen" von der Bundeszentrale für gesundheitliche Aufklärung zur Verfügung (für eine Übersicht siehe Paschke, Austermann \& Thomasius, 2021). Interventionsprogramme richten sich oft ausschließlich an die betroffenen Jugendlichen (z. B. „Lebenslust statt Onlineflucht“; Thomsen, Wartberg \& Thomasius, 2013) oder jungen Erwachsenen (z. B. „STICA“; Wölfling et al., 2019) oder beziehen die Eltern nur am Rande mit ein (z.B. ,Medientraining“; von Wölfling et al., nicht veröffentlicht). Ein familienbasiertes Frühinterventionsprogramm in Deutschland, das die Eltern intensiv mit einbezieht, ist das Programm ESCapade (Thormann \& Tietze, 2019). In diesem Programm werden Gruppen- und Einzelangebote miteinander kombiniert, die sich allesamt an die Familien richten. Das Training konnte die IBS-Symptomatik beim Kind reduzieren und die Familienmitglieder entlasten.

Auch in der internationalen Forschung finden sich nur einzelne Programme, die Eltern intensiv in die Therapie einbeziehen. Q.-X. Liu et al. (2015) zeigten beispielsweise, dass eine eingesetzte Multifamilientherapie (sechs Gruppensitzungen für Jugendliche und für Eltern; Themen: Familienkommunikation, Eltern-Kind-Beziehung, psychologische Bedürfnisse der Jugendlichen) die Rate von Internetabhängigkeit von $100 \%$ auf $4.8 \%$ reduzieren konnte. Die Verbesserungen standen in Zusammenhang mit positiven Veränderungen in der Eltern-Kind-Kommunikation und der Nähe zwischen Eltern und Kind und zeigten sich auch im 3-Monats Follow-up stabil. Han et al.
(2012) berichten, dass Familien mit beeinträchtigter Familienfunktionalität von einem speziellen Training profitierten und sich Bildschirmzeiten der Jugendlichen signifikant reduzierten. In diesem Training wurde u.a. gemeinsame Zeit zwischen Eltern und Kind verordnet (4x/Woche mindestens $1 \mathrm{~h} / \mathrm{Tag})$.

In Tübingen wurde das ISES! Gruppentraining (Internetbezogene Störungen: Eltern stärken!) entwickelt, das im Rahmen von sechs Gruppenterminen Eltern von Jugendlichen und jungen Erwachsenen mit IBS einen Beratungsrahmen bietet, auch wenn der Betroffene selbst nicht motiviert ist, Hilfe in Anspruch zu nehmen. Die Ziele des Trainings sind zum einen, die IBS- oder CS-Symptome durch eine indirekte Einflussnahme durch die Eltern zu reduzieren, und zum anderen, die Eltern zu entlasten und zu stärken. Dies geschieht über eine Anleitung der Eltern zur Verbesserung der Eltern-Kind-Beziehung, Motivation zur gemeinsamen Freizeitgestaltung, Kommunikationstraining, Verbesserung der erzieherischen Kompetenz, Positivierung der Haltung und Einstellung gegenüber der Internetnutzung des Kindes, Verbesserung der Vorbildfunktion sowie über Selbstfürsorgeübungen für die Eltern. Das Trainingsprogramm wird aktuell im Rahmen einer randomisierten kontrollierten Studie evaluiert.

\section{Stärken und Limitationen}

Die Stärken dieser Arbeit bestehen in der Vielfalt der Forschungsarbeiten, die aufgrund der offenen Recherchestrategie einbezogen werden konnten. Dabei gliedert die Arbeit die Bereiche der Eltern-Kind-Beziehung und der Erziehung in weitere Teilbereiche auf und erweitert damit bisherige Übersichtsarbeiten (Schneider et al., 2017; Wartberg et al., 2015). Die meisten Studien beziehen sich auf nichtklinische Schüler- und Studentenstichproben, die Ergebnisse können daher nicht ohne Weiteres auf klinische Stichproben übertragen werden. Es ist davon auszugehen, dass Forschung aus asiatischen Länder, die beispielsweise kollektivistischer geprägt sind, sich nicht ohne Weiteres auf individualistischere Kulturen übertragen lassen (vgl. Garcia, Serra, Garcia, Martinez \& Cruise, 2019). Hier könnte eine systematische Literaturrecherche, welche die Studienergebnisse aus verschiedenen Kulturen der letzten Jahre gegenüberstellt, die Einordnung erleichtern. Eine weitere Limitation ist, dass nur vereinzelt Ergebnisse zu Müttern und Vätern dargestellt wurde, die Hinweise darauf geben, dass Befunde zu den Eltern unterschiedlich sein könnten. Des Weiteren wurde in dieser Übersicht nur das übergeordnete Konstrukt IBS oder Internetsucht beschrieben, nach Möglichkeit spezifiziert nach CS. Dies ist der Situation geschuldet, dass die meisten Studien Instrumente einsetzen, die Internetsucht 
als übergeordnetes Konstrukt beschreiben. Die Ergebnisse können aufgrund auch unterschiedlicher Mechanismen nicht generalisierend auf alle Teilbereiche einer IBS (Computerspielsucht, Sucht nach sozialen Netzwerken, OnlinePornografiesucht, Online-Glücksspielsucht, Online-Kaufsucht) übertragen werden.

Viele Forschungsarbeiten, die für diese Übersichtsarbeit herangezogen wurden, stellen in ihren Ergebnissen nur zusammenfassende Befunde dar, anstatt beispielsweise alle Subskalen eines Fragebogens zur Eltern-Kind-Beziehung im Detail abzubilden (z. B. Vertrauen, erlebte Distanz, Sich-verstanden-Fühlen, die Bereitschaft, sich bei Schwierigkeiten an die Eltern zu wenden; King \& Delfabbro, 2017; X. Wu et al., 2013). Dadurch geht Wissen zu den einzelnen Facetten der Eltern-Kind-Beziehung verloren, das notwendig wäre, um das komplexe Zusammenwirken zwischen Eltern-Kind-Beziehung und IBS besser zu verstehen. Zukünftige Forschungsarbeiten sollten daher mehr Detailergebnisse berichten und mögliche Mediatoren und Moderatoren einbeziehen. Schlussendlich benötigen wir ein multifaktorielles ätiologisches Modell, das die verschiedenen Erkenntnisse der Familienfaktoren integriert und deren Zusammenspiel erkennbar macht und somit zu praxisrelevanten Empfehlungen für Forschung, Prävention und Intervention führt.

\section{Literatur}

Adams, B. L. M., Stavropoulos, V., Burleigh, T.L., Liew, L.W. L., Beard, C. L. \& Griffiths, M. D. (2019). Internet gaming disorder behaviors in emergent adulthood: A pilot study examining the interplay between anxiety and family cohesion. International Journal of Mental Health and Addiction, 17, 828-844. https://doi. org/10.1007/s11469-018-9873-0

American Psychiatric Association. (Ed.). (2013). Diagnostic and Statistical Manual of Mental Disorders (DSM-5), 5th edition. Washington, DC: American Psychiatric Association.

Anderson, E. L., Steen, E. \& Stavropoulos, V. (2017). Internet use and problematic Internet use: A systematic review of longitudinal research trends in adolescence and emergent adulthood. International Journal of Adolescence and Youth, 22, 430-454. https://doi.org/10.1080/02673843.2016.1227716

Appel, M., Holtz, P., Stiglbauer, B. \& Batinic, B. (2012). Parents as a resource: Communication quality affects the relationship between adolescents' point of view Internet use and loneliness. Journal of Adolescence, 35, 1641-1648. https://doi.org/10.10 16/j.adolescence.2012.08.003

Bonnaire, C., Liddle, H.A., Har, A., Nielsen, P. \& Phan, O. (2019). Why and how to include parents in the treatment of adolescents presenting Internet gaming disorder? Journal of Behavioral Addictions, 8, 201-212. https://doi.org/10.1556/2006.8.2019.27

Bonnaire, C. \& Phan, O. (2017). Relationships between parental attitudes, family functioning and Internet gaming disorder in adolescents attending school. Psychiatry Research, 255, 104-110. https://doi.org/10.1016/j.psychres.2017.05.030

Borca, G., Bina, M., Keller, P.S., Gilbert, L. R. \& Begotti, T. (2015). Internet use and developmental tasks: Adolescents' point of view.
Computers in Human Behavior, 52, 49-58. https://doi. org/10.1016/j.chb.2015.05.029

Bussone, S., Trentini, C., Tambelli, R. \& Carola, V. (2020). Early-life interpersonal and affective risk factors for pathological gaming. Frontiers in Psychiatry, 11, 423. https://doi.org/10.3389/ fpsyt.2020.00423

Cacioppo, M., Barni, D., Correale, C., Mangialavori, S., Danioni, F. \& Gori, A. (2019). Do attachment styles and family functioning predict adolescents' problematic Internet use? A relative weight analysis. Journal of Child and Family Studies, 28, 1263-1271. https://doi.org/10.1007/s10826-019-01357-0

Cerniglia, L., Zoratto, F., Cimino, S., Laviola, G., Ammaniti, M. \& Adriani, W. (2017). Internet addiction in adolescence: Neurobiological, psychosocial and clinical issues. Neuroscience and Biobehavioral Reviews, 76(PtA), 174-184.https://doi.org/10.1016/j. neubiorev.2016.12.024

Chen, Y.-L., Chen, S.-H. \& Gau, S.S.-F. (2015). ADHD and autistic traits, family function, parenting style, and social adjustment for Internet addiction among children and adolescents in Taiwan: a longitudinal study. Research in Developmental Disabilities, 39, 20-31. https://doi.org/10.1016/j.ridd.2014.12.025

Chiu, S.-I., Lee, J.-Z. \& Huang, D.-H. (2004). Video game addiction in children and teenagers in Taiwan. Cyber Psychology \& Behavior, 7, 571-581.

Chng, G. S., Li, D., Liau, A. K. \& Khoo, A. (2014). Moderating effects of the family environment for parental mediation and pathological Internet use in youths. Cyberpsychology, Behavior, and Social Networking, 18, 30-36. https://doi.org/10.1089/cyber.2014.0368

Choo, H., Sim, T., Liau, A. K. F., Gentile, D.A. \& Khoo, A. (2015). Parental influences on pathological symptoms of video-gaming among children and adolescents: A prospective study. Journal of Child and Family Studies, 24, 1429-1441. https://doi. org/10.1007/s10826-014-9949-9

Chung, S., Lee, J. \& Lee, H. K. (2019). Personal factors, Internet characteristics, and environmental factors contributing to adolescent Internet addiction: A public health perspective. International Journal of Environmental Research and Public Health, 16, 4635. https://doi.org/10.3390/ijerph16234635

Da Charlie, C.W., HyeKyung, C. \& Khoo, A. (2011). Role of parental relationships in pathological gaming. Procedia - Social and Behavioral Sciences, 30, 1230-1236. https://doi.org/10.1016/j. sbspro.2011.10.238

De Leo, J.A. \& Wulfert, E. (2013). Problematic Internet use and other risky behaviors in college students: An application of problem-behavior theory. Psychology of Addictive Behaviors, 27, 133-141. https://doi.org/10.1037/a0030823

Durkee, T., Kaess, M., Carli, V., Parzer, P., Wasserman, C., Floderus, B. et al. (2012). Prevalence of pathological Internet use among adolescents in Europe: Demographic and social factors. Addiction, 107, 2210-2222. https://doi.org/10.1111/j.1360-0443.201 2.03946.x

Ergün, G. \& Ișık, I. (2018). Determining the relation between Turkish middle- school students' Internet addiction and perceived social support from family. Addicta: The Turkish Journal on Addictions, 5. https://doi.org/10.15805/addicta.2018.5.3.0003

Faltýnková, A., Blinka, L., Ševčíková, A. \& Husarova, D. (2020). The associations between family-related factors and excessive Internet use in adolescents. International Journal of Environmental Research and Public Health, 17, 1754-1765. https://doi. org/10.3390/ijerph17051754

Fidan, A. \& Seferoglu, S. S. (2020). Online environments and digital parenting: An investigation of approaches, problems, and recommended solutions. Bartin University Journal of Faculty of Education, 9, 352-372.

Fumero, A., Marrero, R.J., Voltes, D. \& Peñate, W. (2018). Personal and social factors involved in internet addiction among adole- 
scents: A meta-analysis. Computers in Human Behavior, 86, 387-400. https://doi.org/10.1016/j.chb.2018.05.005

Garcia, F., Serra, E., Garcia, O. F., Martinez, I. \& Cruise, E. (2019). A third emerging stage for the current digital society? Optimal parenting styles in Spain, the United States, Germany, and Brazil. International Journal of Environmental Research and Public Health, 16. https://doi.org/10.3390/ijerph16132333

Gentile, D.A., Choo, H., Liau, A., Sim, T., Li, D., Fung, D. et al. (2011). Pathological video game use among youths: A two-year longitudinal study. Pediatrics, 127, e319-29. https://doi.org/10.1542/ peds.2010-1353

Han, D.h., Kim, S. M., Lee, Y.S. \& Renshaw, P.F. (2012). The effect of family therapy on the changes in the severity of on-line game play and brain activity in adolescents with on-line game addiction. Psychiatry Research, 202, 126-131. https://doi.org/ 10.1016/j.pscychresns.2012.02.011

Hwang, H., Hong, J., Kim, S. M. \& Han, D.h. (2020). The correlation between family relationships and brain activity within the reward circuit in adolescents with Internet gaming disorder. Scientific Reports, 10, 9951. https://doi.org/10.1038/s4159 8-020-66535-3

Iskender, M. (2018). Investigation of the effects of social self-confidence, social loneliness and family emotional loneliness variables on Internet addiction. Malaysian Online Journal of Educational Technology, 6(3), 1-10. https://doi.org/10.17220/mojet. 2018.03.001

Jang, M.H. \& Ji, E.S. (2012). Gender differences in associations between parental problem drinking and early adolescents' internet addiction. Journal for Specialists in Pediatric Nursing, 17, 288-300. https://doi.org/10.1111/j.1744-6155.2012.00344.x

Jeong, E.J. \& Kim, D.h. (2011). Social activities, self-efficacy, game attitudes, and game addiction. Cyberpsychology, Behavior, and Social Networking, 14, 213-221. https://doi.org/10.1089/cy ber.2009.0289

Kalmus, V., Blinka, L. \& Olafsson, K. (2015). Does it matter what mama says: Evaluating the role of parental mediation in European adolescents' excessive Internet use.Children @ Society, 29, 122-133.

Kammerl, R., Hirschhäuser, L., Rosenkranz, M., Schwinge, C., Hein, S., Wartberg, L. et al. (2012). Exzessive Internetnutzung in Familien - Zusammenhänge zwischen der exzessiven Computer- und Internetnutzung Jugendlicher und dem (medien)erzieherischen Handeln in den Familien. Lengerich: Pabst Science.

Kammerl, R. \& Wartberg, L. (2018). Zusammenhänge zwischen problematischer Internetnutzung im Jugendalter und Medienerziehung in der Familie. Praxis der Kinderpsychologie und Kinderpsychiatrie, 67, 134-153.

Karaer, Y. \& Akdemir, D. (2019). Parenting styles, perceived social support and emotion regulation in adolescents with Internet addiction. Comprehensive Psychiatry, 92, 22-27. https://doi. org/10.1016/j.comppsych.2019.03.003

Kerr, M., Stattin, H. \& Burk, W.J. (2010). A reinterpretation of parental monitoring in longitudinal perspective. Journal of Research on Adolescence, 20, 39-64. https://doi.org/10.1111/j.1532-7 795.2009.00623.x

Kim, K. \& Kim, K. (2015). Internet game addiction, parental attachment, and parenting of adolescents in South Korea. Journal of Child \& Adolescent Substance Abuse, 24, 366-371. https://doi. org/10.1080/1067828X.2013.872063

King, D. L. \& Delfabbro, P.H. (2017). Features of parent-child relationships in adolescents with Internet gaming disorder. International Journal of Mental Health and Addiction, 15, 1270-1283. https://doi.org/10.1007/s11469-016-9699-6

Ko, C.-H., Yen, J.-Y., Yen, C.-F., Lin, H.-C. \& Yang, M.-J. (2007). Factors predictive for incidence and remission of Internet addiction in young adolescents: A prospective study. Cyberpsychology \&
Behavior: The Impact of the Internet, Multimedia and Virtual Reality on Behavior and Society, 10, 545-551. https://doi. org/10.1089/cpb.2007.9992

Kraut, R., Patterson, M., Lundmark, V., Kiesler, S., Mukophadhyay, T. \& Scherlis, W. (1998). Internet paradox: A social technology that reduces social involvement and psychological well-being? American Psychologist, 53, 1017-1031.

Kveton, P. \& Jelinek, M. (2016). Parenting styles and their relation to videogame addiction. International Journal of Psychological and Behavioral Sciences, 10, 1961-1964.

Kwon, J.-H., Chung, C.-S. \& Lee, J. (2011). The effects of escape from self and interpersonal relationship on the pathological use of Internet games. Community Mental Health Journal, 47 , 113-121. https://doi.org/10.1007/s10597-009-9236-1

Lam, L.T. (2014). Risk factors of Internet addiction and the health effect of internet addiction on adolescents: A systematic review of longitudinal and prospective studies. Current Psychiatry Reports, 16, 508. https://doi.org/10.1007/s11920-014-0508-2

Lam, L.T. (2015). Parental mental health and Internet addiction in adolescents. Addictive Behaviors, 42, 20-23. https://doi.org/ 10.1016/j.addbeh.2014.10.033

Lam, L.T. (2020). The roles of parent-and-child mental health and parental Internet addiction in adolescent Internet addiction: Does a parent-and-child gender match matter? Frontiers in $\mathrm{Pu}$ blic Health, 8, 142. https://doi.org/10.3389/fpubh.2020.00142

Lam, L.T. \& Wong, E. M.Y. (2015). Stress moderates the relationship between problematic Internet use by parents and problematic Internet use by adolescents. Journal of Adolescent Health: Official Publication of the Society for Adolescent Medicine, 56, 300-306. https://doi.org/10.1016/j.jadohealth.2014.10.263

Lee, S.-J. \& Chae, Y.-G. (2007). Children's Internet use in a family context: Influence on family relationships and parental mediation. Cyberpsychology \& Behavior: The Impact of the Internet, Multimedia and Virtual Reality on Behavior and Society, 10, 640644. https://doi.org/10.1089/cpb.2007.9975

Li, X., Li, D. \& Newman, J. (2013). Parental behavioral and psychological control and problematic Internet use among Chinese adolescents: The mediating role of self-control. Cyberpsychology, Behavior, and Social Networking, 16, 442-447. https://doi.org/ 10.1089/cyber.2012.0293

Liau, A. K., Choo, H., Li, D., Gentile, D.A., Sim, T. \& Khoo, A. (2015). Pathological video-gaming among youth: A prospective study examining dynamic protective factors. Addiction Research \& Theory, 23, 301-308. https://doi.org/10.3109/16066359.2014.9 87759

Lin, C.-H., Lin, S.-L. \& Wu, C.-P. (2009). The effects of parental monitoring and leisure boredom on adolescents' Internet addiction. Adolescence, 44, 993-1004.

Lin, Y.-H. \& Gau, S. S.-F. (2013). Association between morningnesseveningness and the severity of compulsive Internet use: The moderating role of gender and parenting style. Sleep Medicine, 14,1398-1404. https://doi.org/10.1016/j.sleep.2013.06.015

Liu, C.-Y. \& Kuo, F.-Y. (2007). A study of Internet addiction through the lens of the interpersonal theory. Cyberpsychology \& Behavior: The Impact of the Internet, Multimedia and Virtual Reality on Behavior and Society, 10, 799-804. https://doi.org/10.1089/ cpb.2007.9951

Liu, Q.-X., Fang, X.-Y., Deng, L.-Y. \& Zhang, J.-T. (2012). Parent-adolescent communication, parental Internet use and Internetspecific norms and pathological Internet use among Chinese adolescents. Computers in Human Behavior, 28(4), 1269-1275. https://doi.org/10.1016/j.chb.2012.02.010

Liu, Q.-X., Fang, X.-Y., Yan, N., Zhou, Z.-K., Yuan, X.-J., Lan, J. et al. (2015). Multi-family group therapy for adolescent Internet addiction: Exploring the underlying mechanisms. Addictive Behaviors, 42, 1-8. https://doi.org/10.1016/j.addbeh.2014.10.021 
Liu, Q.-X., Fang, X.-Y., Zhou, Z.-K., Zhang, J.-T. \& Deng, L.-Y. (2013). Perceived parent-adolescent relationship, perceived parental online behaviors and pathological internet use among adolescents: Gender-specific differences. PloS One, 8, e75642. https://doi.org/10.1371/journal.pone.0075642

Männikkö, N., Ruotsalainen, H., Miettunen, J., Pontes, H. M. \& Kääriäinen, M. (2020). Problematic gaming behaviour and healthrelated outcomes: A systematic review and meta-analysis. Journal of Health Psychology, 25, 67-81.

Martins, M.V., Formiga, A., Santos, C., Sousa, D., Resende, C., Campos, R. et al. (2020). Adolescent internet addiction - role of parental control and adolescent behaviours. International Journal of Pediatrics \& Adolescent Medicine, 7, 116-120. https://doi. org/10.1016/j.ijpam.2019.12.003

Medienpädagogischer Forschungsverbund Südwest. (Hrsg.). (2017). FIM-Studie 2016: Familie, Interaktion, Medien. Untersuchung zur Kommunikation und Mediennutzung in Familien. Stuttgart.

Mesch, G.S. (2009). Parental mediation, online activities, and cyberbullying. Cyberpsychology \& Behavior: The Impact of the Internet, Multimedia and Virtual Reality on Behavior and Society, 12,387-393. https://doi.org/10.1089/cpb.2009.0068

Mihara, S. \& Higuchi, S. (2017). Cross-sectional and longitudinal epidemiological studies of Internet gaming disorder: A systematic review of the literature. Psychiatry and Clinical Neurosciences, 71, 425-444. https://doi.org/10.1111/pcn.12532

Oh, W.O. (2003). Factors influencing internet addiction tendency among middle school students in Gyeong-buk area. Journal of Korean Academy of Nursing, 33, 1135-1144.

Padilla-Walker, L.M. \& Coyne, S.M. (2011). „Turn that thing off! “ Parent and adolescent predictors of proactive media monitoring. Journal of Adolescence, 34, 705-715. https://doi. org/10.1016/j.adolescence.2010.09.002

Park, S. K., Kim, J.Y. \& Cho, C. B. (2008). Prevalence of Internet addiction and correlations with family factors among South Korean adolescents. Adolescence, 43, 895-909.

Paschke, K., Austermann, M.I. \& Thomasius, R. (2021). Assessing ICD-11 gaming disorder in adolescent gamers by parental ratings: Development and validation of the Gaming Disorder Scale for Parents (GADIS-P). Journal of Behavioral Addictions, 10, 159-168. https://doi.org/10.1556/2006.2020.00105

Rehbein, F. \& Baier, D. (2013). Family-, media-, and school-related risk factors of video game addiction. Journal of Media Psychology, 25, 118-128. https://doi.org/10.1027/1864-1105/a000093

Richards, R., McGee, R., Williams, S.M., Welch, D. \& Hancox, R.J. (2010). Adolescent screen time and attachmentto parents and peers. Archives of Pediatrics and Adolescent Medicine, 164 , 258-262.

Rikkers, W., Lawrence, D., Hafekost, J. \& Zubrick, S. R. (2016). Internet use and electronic gaming by children and adolescents with emotional and behavioural problems in Australia - results from the second Child and Adolescent Survey of Mental Health and Wellbeing. BMC Public Health, 16, 399. https://doi.org/10.1186/ s12889-016-3058-1

Rumpf, H.-J., Arnaud, N., Batra, A., Bischof, A., Bischof, G., Brand, M. et al. (2016). Memorandum Internetbezogene Störungender Deutschen Gesellschaft für Suchtforschung und Suchttherapie (DG-Sucht). Sucht, 62, 167-172.

Sanders, C. E., Field, T.M., Diego, M. \& Kaplan, M. (2000). The relationship of Internet use to depression and social isolation among adolescents. Adolescence, 35, 237.

Schimmenti, A., Passanisi, A., Gervasi, A. M., Manzella, S. \& Famà, F.I. (2014). Insecure attachment attitudes in the onset of problematic Internet use among late adolescents. Child Psychiatry and Human Development, 45, 588-595. https://doi.org/10.1007/ s10578-013-0428-0
Schneider, L.A., King, D.L. \& Delfabbro, P.H. (2017). Family factors in adolescent problematic Internet gaming: A systematic review. Journal of Behavioral Addictions, 6, 321-333. https://doi. org/10.1556/2006.6.2017.035

Shek, D.T.L. \& Yu, L. (2016). Adolescent Internet addiction in Hong Kong: Prevalence, change, and correlates. Journal of Pediatric and Adolescent Gynecology, 29(1 Suppl), S22-30. https://doi. org/10.1016/j.jpag.2015.10.005

Shek, D.T.L., Zhu, X. \& Dou, D. (2019). Influence of family processes on internet addiction among late adolescents in Hong Kong. Frontiers in Psychiatry, 10, 113. https://doi.org/10.3389/fps yt. 2019.00113

Shi, X., Wang, J. \& Zou, H. (2017). Family functioning and Internet addiction among Chinese adolescents: The mediating roles of self-esteem and loneliness. Computers in Human Behavior, 76, 201-210. https://doi.org/10.1016/j.chb.2017.07.028

Siomos, K., Floros, G., Fisoun, V., Evaggelia, D., Farkonas, N., Sergentani, E. et al. (2012). Evolution of Internet addiction in Greek adolescent students over a two-year period: The impact of parental bonding. European Child \& Adolescent Psychiatry, 21, 211-219. https://doi.org/10.1007/s00787-012-0254-0

Siste, K., Hanafi, E., Jamtani, D., Murtani, B.J., Beatrice, E., Christian, H. et al. (2020). Gaming disorder and parenting style: A case series. Addictive Disorders \& Their Treatment, 19, 185-190. https://doi.org/10.1097/ADT.0000000000000195

Stevens, M.W.R., King, D.L., Dorstyn, D. \& Delfabbro, P.H. (2019). Cognitive-behavioral therapy for Internet gaming disorder: A systematic review and meta-analysis. Clinical Psychology \& Psychotherapy, 26, 191-203. https://doi.org/10.1002/cpp.2341

Su, B., Yu, C., Zhang, W., Su, Q., Zhu, J. \& Jiang, Y. (2018). Fatherchild longitudinal relationship: parental monitoring and Internet gaming disorder in Chinese adolescents. Frontiers in Psychology, 9, 95. https://doi.org/10.3389/fpsyg.2018.00095

Sugaya, N., Shirasaka, T., Takahashi, K. \& Kanda, H. (2019). Biopsychosocial factors of children and adolescents with internet gaming disorder: A systematic review. BioPsychoSocial Medicine, 13, 3. https://doi.org/10.1186/s13030-019-0144-5

Szasz-Janocha, C., Kindt, S., Halasy, K. \& Lindenberg, K. (2019). Prävention und Frühintervention bei Internet-bezogenen Störungen - (inter-)nationaler Stand der Forschung. Suchtmedizin, 21, 259-271.

Tafà, M. \& Baiocco, R. (2009). Addictive behavior and family functioning during adolescence. American Journal of Family Therapy, 37, 388-395. https://doi.org/10.1080/01926180902754745

Thomsen, M., Wartberg, L. \& Thomasius, R. (2013). Die Behandlung von pathologischem Internetgebrauch bei Jugendlichen - Pilotdaten zur Wirksamkeit eines ambulanten Gruppenprogramms. Suchttherapie, 14(S 01), S_50_2.

Thormann, J. \& Tietze, K. (2019). ESCapade: Interventionsprogramm für Familien und Kinder mit problematischer Mediennutzung. In S. Rietmann, M. Sawatzki \& M. Berg (Hrsg.), Beratung und Digitalisierung (Bd. 15, S. 341-352). Wiesbaden: Springer Fachmedien Wiesbaden.

Throuvala, M.A., Janikian, M., Griffiths, M.D., Rennoldson, M. \& Kuss, D.J. (2019). The role of family and personality traits in Internet gaming disorder: A mediation model combining cognitive and attachment perspectives. Journal of Behavioral Addictions, 8, 48-62. https://doi.org/10.1556/2006.8.2019.05

Tóth-Király, I., Morin, A.J.S., Hietajärvi, L. \& Salmela-Aro, K. (2021). Longitudinal trajectories, social and individual antecedents, and outcomes of problematic Internet use among late adolescents. Child Development. https://doi.org/10.1111/cdev.13525

Tsitsika, A., Critselis, E., Louizou, A., Janikian, M., Freskou, A., Marangou, E. et al. (2011). Determinants of Internet addiction among adolescents: A case-control study. TheScientificWorldJournal, 11, 866-874. https://doi.org/10.1100/tsw.2011.85 
Vadlin, S., Åslund, C., Hellström, C. \& Nilsson, K.W. (2016). Associations between problematic gaming and psychiatric symptoms among adolescents in two samples. Addictive Behaviors, 61 8-15. https://doi.org/10.1016/j.addbeh.2016.05.001

Valcke, M., Bonte, S., de Wever, B. \& Rots, I. (2010). Internet parenting styles and the impact on Internet use of primary school children. Computers \& Education, 55(2), 454-464. https://doi. org/10.1016/j.compedu.2010.02.009

van den Eijnden, R.J.J.M., Spijkerman, R., Vermulst, A.A., van Rooij, T.J. \& Engels, R.C.M.E. (2010). Compulsive internet use among adolescents: Bidirectional parent-child relationships. Journal of Abnormal Child Psychology, 38, 77-89. https://doi. org/10.1007/s10802-009-9347-8

van Petegem, S., de Ferrerre, E., Soenens, B., van Rooij, A.J. \& van Looy, J.(2019). Parents' degree and style of restrictive mediation of young children's digital gaming: associations with parental attitudes and perceived child adjustment. Journal of Child and Family Studies, 28, 1379-1391. https://doi.org/10.1007/s10826-019-01368-x Venkatesh, V., Sykes, T.A., Chan, F.K.Y., Thong, J.Y.L. \& Hu, P.J.-H. (2019). Children's Internet addiction, family-to-work conflict, and job outcomes: a study of parent-child dyads. MIS Q, 43(3), 903-928. https://doi.org/10.25300/MISQ/2019/12338

Vondráčková, P. \& Gabrhelík, R. (2016). Prevention of Internet addiction: A systematic review. Journal of behavioral addictions, 5, 568-579. https://doi.org/10.1556/2006.5.2016.085

Wang, C.-W., Chan, C. L.W., Mak, K.-K., Ho, S.-Y., Wong, P.W.C. \& Ho, R.T.H. (2014). Prevalence and correlates of video and Internet gaming addiction among Hong Kong adolescents: A pilot study. Scientific World Journal, 2014, 874648. https://doi.org/10. $1155 / 2014 / 874648$

Wang, W., Li, D., Li, X., Wang, Y., Sun, W., Zhao, L. et al. (2018). Parent-adolescent relationship and adolescent Internet addiction: A moderated mediation model. Addictive Behaviors, 84, 171-177. https://doi.org/10.1016/j.addbeh.2018.04.015

Wartberg, L., Aden, A., Thomsen, M. \& Thomasius, R. (2015). Zusammenhänge zwischen familialen Aspekten und pathologischem Internetgebrauch bei Jugendlichen. Zeitschrift fur Kinder- und Jugendpsychiatrie und Psychotherapie, 43, 9-17; quiz 18-19. https://doi.org/10.1024/1422-4917/a000328

Wartberg, L., Kriston, L., Kramer, M., Schwedler, A., Lincoln, T.M. \& Kammerl, R. (2017). Internet gaming disorder in early adolescence: Associations with parental and adolescent mental health. European Psychiatry: The Journal of the Association of European Psychiatrists, 43, 14-18. https://doi.org/10.1016/j. eurpsy.2016.12.013

Wartberg, L., Zieglmeier, M. \& Kammerl, R. (2019). Accordance of adolescent and parental ratings of Internet gaming disorder and their associations with psychosocial aspects. Cyberpsychology, Behavior, and Social Networking, 22, 264-270. https:// doi.org/10.1089/cyber.2018.0456

Wölfling, K., Müller, K.W., Dreier, M., Ruckes, C., Deuster, O., Batra, A. et al. (2019). Efficacy of short-term treatment of Internet and computer game addiction: A randomized clinical trial. JAMA Psychiatry, 76(10), 1018-1025. https://doi.org/10.1001/jama psychiatry.2019.1676

Wu, C.S.T., Wong, H.T., Yu, K. F., Fok, K. W., Yeung, S. M., Lam, C. H. et al. (2016). Parenting approaches, family functionality, and internet addiction among Hong Kong adolescents. BMC Pediatrics, 16, 130. https://doi.org/10.1186/s12887-016-0666-y

Wu, J.Y.W., Ko, H.-C., Wong, T.-Y., Wu, L.-A. \& Oei, T.P. (2016). Positive Outcome Expectancy Mediates the relationship between peer influence and Internet gaming addiction among adolescents in Taiwan. Cyberpsychology, Behavior, and Social Networking, 19, 49-55. https://doi.org/10.1089/cyber.2015.0345

Wu, X., Chen, X., Han, J., Meng, H., Luo, J., Nydegger, L. et al. (2013). Prevalence and factors of addictive Internet use among adole- scents in Wuhan, China: Interactions of parental relationship with age and hyperactivity-impulsivity. PloS One, 8, e61782. https://doi.org/10.1371/journal.pone.0061782

Wu, X.-S., Zhang, Z.-H., Zhao, F., Wang, W.-J., Li, Y.-F., Bi, L. et al. (2016). Prevalence of Internet addiction and its association with social support and other related factors among adolescents in China. Journal of Adolescence, 52, 103-111. https://doi. org/10.1016/j.adolescence.2016.07.012

Xin, M., Xing, J., Pengfei, W., Houru, L., Mengcheng, W. \& Hong, Z. (2018). Online activities, prevalence of Internet addiction and risk factors related to family and school among adolescents in China. Addictive Behaviors Reports, 7, 14-18. https://doi. org/10.1016/j.abrep.2017.10.003

Xiuquin, H., Huimin, Z., Mengchen, L., Jinan, W., Ying, Z. \& Ran, T. (2010). Mental health, personality, and parental rearing stylesof adolescents with Internet addiction disorder. Cyberpsychology, Behavior, and Social Networking, 13, 401-406.

Xu, J., Shen, L., Yan, Chong-huai, Hu, Howard, Yang, F., Wang, L., Kotha, S. R. et al. (2014). Parent-adolescent interaction and risk of adolescent internet addiction: A population-based study in Shanghai. BMC Psychiatry, 14(112), 1-11.

Xu, Z., Turel, O. \& Yuan, Y. (2012). Online game addiction among adolescents: Motivation and prevention factors. European Journal of Information Systems, 21, 321-340. https://doi. org/10.1057/ejis.2011.56

Yang, X., Zhu, L., Chen, Q., Song, P. \& Wang, Z. (2016). Parent marital conflict and Internet addiction among Chinese college students: The mediating role of father-child, mother-child, and peer attachment. Computers in Human Behavior, 59, 221-229. https://doi.org/10.1016/j.chb.2016.01.041

Yen, C.-F., Ko, C.-H., Yen, J.-Y., Chang, Y.-P. \& Cheng, C.-P. (2009). Multi-dimensional discriminative factors for Internet addiction among adolescents regarding gender and age. Psychiatry and Clinical Neurosciences, 63, 357-364. https://doi.org/10.1111/ j.1440-1819.2009.01969.x

Yen, J.-Y., Yen, C.-F., Chen, C.-C., Chen, S.-H. \& Ko, C.-H. (2007). Family factors of internet addiction and substance use experience in Taiwanese adolescents. Cyberpsychology \& Behavior: The Impact of the Internet, Multimedia and Virtual Reality on Behavior and Society, 10, 323-329. https://doi.org/10.1089/cpb.2006.9948

Yu, L. \& Shek, D.T.L. (2013). Internet addiction in Hong Kong adolescents: A three-year longitudinal study. Journal of Pediatric and Adolescent Gynecology, 26(3 Suppl), S10-7. https://doi. org/10.1016/j.jpag.2013.03.010

Zajac, K., Ginley, M.K., Chang, R. \& Petry, N. M. (2017). Treatments for Internet gaming disorder and Internet addiction: A systematic review. Psychology of Addictive Behaviors: Journal of the Society of Psychologists in Addictive Behaviors, 31, 979-994. https://doi.org/10.1037/adb0000315

Zhang, H., Luo, Y., Yao, Z. \& Barrow, K. (2021). The role of resting respiratory sinus arrhythmia in the family functioning-internet addiction symptoms link. International Journal of Psychophysiology, 164,17-22. https://doi.org/10.1016/j.ijpsycho.2021.02.012

Zhang, H., Spinrad, T.L., Eisenberg, N., Luo, Y. \& Wang, Z. (2017). Young adults' internet addiction: Prediction by the interaction of parental marital conflict and respiratory sinus arrhythmia. International Journal of Psychophysiology: Official Journal of the International Organization of Psychophysiology, 120, 148-156. https://doi.org/10.1016/j.ijpsycho.2017.08.002

Zhu, J., Zhang, W., Yu, C. \& Bao, Z. (2015). Early adolescent Internet game addiction in context: How parents, school, and peers impact youth. Computers in Human Behavior, 50, 159-168. https:// doi.org/10.1016/j.chb.2015.03.079

Zorbas, S.D., Ulas, O. \& Kizildag, S. (2015). Relationship between video game addiction and interfamiliy relationships on primary. Educational Sciences: Theory \& Practice, 15, 489-497. 
Historie

Manuskript eingereicht: 28.02.2021

Nach Revision angenommen: 21.05.2021

Onlineveröffentlichung: 09.07.2021

\section{Interessenkonflikte}

Das Ministerium für Soziales und Integration Baden-Württemberg fördert das themennahe Forschungsprojekt ,Internetbezogene Störungen: Eltern stärken - ISES!“. Alle drei Autoren sind an diesem Forschungsprojekt beteiligt. Interessenkonflikte bestehen für alle Autoren jedoch nicht.

\section{Förderung}

Open-Access-Veröffentlichung ermöglicht durch die Eberhard Karls Universität Tübingen.
Dr. Dipl.-Psych. Isabel Brandhorst

Universitätsklinikum Tübingen

Klinik für Psychiatrie und Psychotherapie

Abteilung Psychiatrie, Psychosomatik und Psychotherapie im

Kindes- und Jugendalter

Osianderstr. 14

72076 Tübingen

Deutschland

isabel.brandhorst@med.uni-tuebingen.de

\section{CME-Fragen}

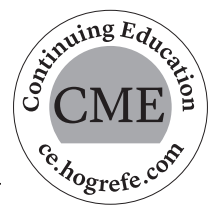

1. Frage: Für welchen Aspekt der folgenden Familienfaktoren liegen die meisten Studienergebnisse in Bezug auf Internetbezogenen Störungen bei Jugendlichen vor? (Einfachauswahl)

a. Eltern-Kind-Beziehung

b. Erziehungsverhalten

c. Kontextfaktoren

d. Familienkommunikation

e. Belastungsfaktoren

\section{Frage: Welcher Aussage kann man nicht} zustimmen: (Einfachauswahl)

a. Es gibt positive Zusammenhänge zwischen negativer Familienkommunikation und Symptomen einer Internetbezogenen Störung bei Jugendlichen.

b. Es gibt positive Zusammenhänge zwischen Familienkonflikten und Symptomen einer Internetbezogenen Störung bei Jugendlichen.

c. Es gibt positive Zusammenhänge zwischen Ermahnen und Warnen vor Computerspielen und Symptomen einer Internetbezogenen Störung bei Jugendlichen.

d. Eine schlechte Qualität der Kommunikation gilt als Risikofaktor für die spätere Entwicklung einer Internetbezogenen Störung bei Jugendlichen.

e. Eine geringe Quantität der Kommunikation gilt als Risikofaktor für die spätere Entwicklung einer Internetbezogenen Störung bei Jugendlichen. c. 3-fach erhöht

d. 4 -fach erhöht

e. 5 -fach erhöht

\section{Frage: Welcher Aussage kann man aufgrund} der vorliegend Forschungslage klar zustimmen?

(Einfachauswahl)

a. Regeln und Restriktionen schützen Jugendliche vor der Entwicklung einer Internetbezogenen Störung.

b. Regeln und Restriktionen fördern Internetbezogene Störungen bei Jugendlichen.

c. Regeln und Restriktionen haben keinen Einfluss auf Internetbezogene Störungen bei Jugendlichen.

d. Die Forschung zeigt kein einheitliches Bild zur Wirkung von Regeln und Restriktionen auf Internetbezogene Störungen bei Jugendlichen.

e. Regeln und Restriktionen sind in der Erziehung grundsätzlich schädlich.

\section{Frage: Welches Interventionsprogramm richtet} sich an Eltern von Kindern oder Jugendlichen mit Internetbezogenen Störungen: (Einfachauswahl)
a. Lebenslust statt Onlineflucht
b. ISES Gruppentraining
c. STICA
d. Medientraining
e. ins-netz-gehen

\section{Frage: Wie stark erhöht ist das Risiko, dass Kinder} eine Internetbezogene Störung entwickeln, wenn die Eltern bereits eine solche Symptomatik zeigen?

(Einfachauswahl)

a. 1-fach erhöht

b. 2-fach erhöht 
Um Ihr CME-Zertifikat zu erhalten (min. drei richtige Antworten), schicken Sie bitte den ausgefüllten Fragebogen mit einem frankierten Rückumschlag bis zum 27.02.2022 an die nebenstehende Adresse. Später eintreffende Antworten und solche ohne bzw. mit nicht frankierten Rückumschlägen können nicht mehr berücksichtigt werden.

\section{Milena Becker}

LWL-Universitätsklinik Hamm der Ruhr-Universität Bochum Klinik für Kinder- und Jugend psychiatrie, Psychotherapie und Psychosomatik

Heithofer Allee 64

59071 Hamm, Deutschland

\section{Fortbildungszertifikat}

Die Ärztekammer Niedersachsen erkennt hiermit

2 Fortbildungspunkte an.

\section{Stempel \\ Kinder- und Jugend- psychiatrie und Psychotherapie \\ $01 / 2022$ \\ (v) hogrefe}

Wichtig: Bitte kleben Sie hier Ihr Barcode-

Etikett (EFN) an oder schreiben Sie Ihre EFN.

Bitte teilen Sie uns alternativ Ihre

Psychotherapeuten-Fortbildungsnummer mit:

Datum

Unterschrift
„Elternfaktoren bei Internet- und Computerspielsucht im Jugendalter"

Die Antworten bitte deutlich ankreuzen! a
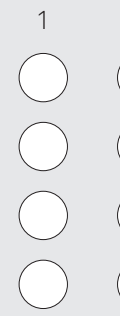

e

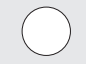

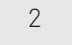

0
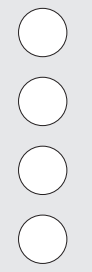

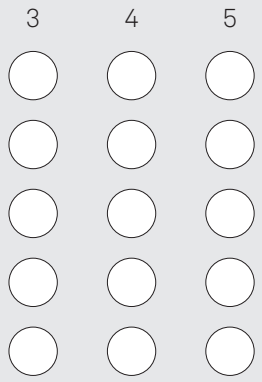

Ich versichere, alle Fragen ohne fremde Hilfe beantwortet zu haben.

Name

Berufsbezeichnung, Titel

Straße, Nr.

PLZ, Ort 